\title{
Synthesis of Ru(bpy) ${ }_{3}$-doped Silica Nanoparticle and Its Application in Fluorescent Immunoassay
}

\author{
Dongguang Yin ${ }^{1 *}$, Binhu Liu, Li Zhang, Chunjuan Xie, Le Zhang \\ ${ }^{1}$ College of Environmental and Chemical Engineering, Shanghai University, Shanghai \\ *Corresponding authors. Email: ydg@shu.edu.cn
}

\begin{abstract}
A novel type of amino functionalized core-shell Ru(bpy) ${ }_{3}$-doped silica nanoparticles was synthesized using a simple and effective approach of reverse microemulsion. The nanoparticles were characterized by transmission electron microscope, fluorescence spectra, UV-Vis spectroscopy and tests of photostability and dye molecular leakage. It was found that the nanoparticles exhibit excellent fluorescent properties such as extremely bright, highly photostable and chemicalstable. Furthermore, the nanoparticles utilized as a fluorescent marker applying in fluorescent immunoassay of mouse IgG were studied and desired results were obtained.
\end{abstract}

Keywords: Ru(bpy) ${ }_{3}$-doped; Silica nanoparticles ; Reverse microemulsion; Immunoassay

Citation: D. Yin et al.. Study on preparation of Ru(bpy)3-doped silica nanoparticles and their application in fluorescent immunoassay. Nano Biomed Eng. 2010, 2(2), 117-120. DOI: 10.5101/nbe.v2i2.p117-120.

\section{Introduction}

Currently, nanoparticles-based techniques shows great promise in applications of bioassay and biomedical [1-6]. Dye-doped silica nanoparticle have many unique properties such as highly fluorescent intensity, highly photostability, and excellent biological compatibility $[7,8]$. Caused on the fact of these, preparation of new silica nanoparticles internally doped with organic and inorganic fluorophores and their application in biological detection and immunoassay were widely investigated and effectively approached.

In this study, we synthesized dye-doped nanoparticles using a water-in-oil microemulsion method by doping the fluorescent dye of tris (2'2-bipyridyl) dichlororuthenium (II) hexahydrate $\left(\mathrm{Ru}(\mathrm{bpy})_{3}\right)$ inside silica material. Approach of water-in-oil microemulsion has advantages of no requirement of extreme condition such as temperature and pressure. In addition, size and shape of the nanoparticles can be simply controlled by varying microemulsion parameters. A silane reagent of 3-aminopropyl-trimethoxysilane (APTMS) was first time used to prepare the nanoparticles. It directly introduced primary amino groups to surface of the nanopar- ticles by copolymerization reaction with tetraethyl orthosilicate (TEOS) [9]. After conjugating with streptavidin, immunoassay experiments using the nanoparticles as fluorescent probe were performed.

\section{Materials and Methods}

\subsection{Materials and Instruments}

$\mathrm{Ru}(\mathrm{bpy})_{3}$, TritonX-100, mouse IgG, goat anti-mouse IgG, BSA, strepavidin (SA) were purchased from sigma. TEOS, APTMS, n-hexanol, cyclohexane and ammonium hydroxide, (28-30 wt \%) were purchased from Shanghai Chemical Reagent Co.Ltd. (Shanghai, China). Ultrapure water (18 M $\Omega$ ) was used for the preparation of all aqueous solutions. Unless otherwise stated, all chemicals and reagents used in this study were analytical grade quality.

Hitachi 800 transmission electron micro-scope(Japan) was used for measuring shape and size of nanoparticles. UV-Vis absorption spectra were recorded on a Hitachi U-3010 UV-Vis spectro-photometer (Japan). Fluorescence spectra were recorded on a Hitachi F-7000 spectrofluorometer (Japan). Fluorescence immunoassay was carried out with Corning FluoroNunc 96-well mi- 
crotiter plate as the solid-phase carrier and measured on a Thermo flash multimode microplate reader with 452nm of excitation wavelength, $608 \mathrm{~nm}$ of emission wavelength.

\subsection{Preparation of $\mathbf{R u}(\mathrm{bpy})_{3}$-doped nanoparticles}

Nanoparticles were synthesized by using a microemulsion method as Santra et al described [10] previously. Firstly, the microemulsion solution was prepared by mixing cyclohexane $(7.5 \mathrm{~mL})$, n-hexanol (1.8mL), Triton X-100 $(1.77 \mu \mathrm{L})$, and water (400ul) with vigorous stirring. After mixing for $20 \mathrm{~min}, 0.5 \mathrm{mg}$ $\mathrm{Ru}(\mathrm{bpy}) 3$ dye in water(0.1mL), $100 \mu \mathrm{L}$ TEOS and $5 \mu \mathrm{L}$ APTMS were added to the mixture. After stirring for $20 \mathrm{~min}, 60 \mu \mathrm{L}$ of $\mathrm{NH}_{4} \mathrm{OH}$ was added to initiate the polymerization reaction. The reaction was allowed to continue for $24 \mathrm{~h}$. When the polymerization was complete, an equal volume of acetone was added, and the mixture was vortexed to break the microemulsion state. The solidified silica nanoparticles were collected by centrifugation at $12000 \mathrm{r} / \mathrm{min}$ for $10 \mathrm{~min}$ and ultrasonically washed with water and $95 \%$ ethanol two times to remove residual surfactant and dye molecules, respectively. After air drying, desirable products of the nanoparticles were obtained.

\subsection{Photobleaching experiments and dyeleaking ex- periments}

To evaluate photostability of the nanoparticles, photobleaching experiments of the nanoparticles and pure $\mathrm{Ru}(\mathrm{bpy})_{3}$ were performed in aqueous solution using a $150 \mathrm{~W}$ xenon lamp as an excitation source. The nanoparticles were continuously exposed at $452 \mathrm{~nm}$ of maximum excitation wavelength. Fluorescent intensities of the nanoparticles were recorded at every $15 \mathrm{~min}$ interval for a period of $1.5 \mathrm{~h}$ on a spectrfluorometer with 591 $\mathrm{nm}$ of emission wavelength.

To perform dyeleaking experiments, $1 \mathrm{mg}$ of the nanoparticles was dissolved in $10 \mathrm{~mL}$ water and the mixture was ultrasonic dispersed continuously. At every $1 \mathrm{~h}$, $1 \mathrm{~mL}$ of the suspending solution was taken out and centrifugated. After centrifugal separation, the precipitate was redissolved in $1 \mathrm{ml}$ water, ultrasonic dispersed, and then emission spectra were recorded. According to varies of the fluorescent intensity with time, dyeleaking of the nanoparticles in aqueous solution was evaluated.

\subsection{Preparation of nanoparticles-labeled SA}

To conjugate with SA [11], the nanoparticles were coated with BSA first. 1.0mg nanoparticles ultrasonic dispersed in $1.0 \mathrm{~mL}$ of $0.1 \mathrm{M}$ phosphate buffer $(\mathrm{pH} 7.0)$ was mixed with $4.0 \mathrm{mg}$ of BSA and $0.3 \mathrm{~mL}$ of $1 \%(\mathrm{v} / \mathrm{v})$ glutaraldehyde, stirred for $24 \mathrm{~h}$ at $4^{\circ} \mathrm{C}$. After centrifug- ing and washing with the phosphate buffer two times, the BSA coated nanoparticles were suspended in $1.0 \mathrm{~mL}$ of the phosphate buffer again, then $200 \mu \mathrm{g}$ of SA and $0.2 \mathrm{~mL}$ of $1 \%$ glutaraldehyde were added. After stirring at $4^{\circ} \mathrm{C}$ for $24 \mathrm{~h}, 2 \mathrm{mg}$ of $\mathrm{NaBH}_{4}$ was added, the reaction was allowed to continue for $2 \mathrm{~h}$. After being centrifuged and washed with the phosphate buffer and water, the nanoparticle-labeled SA was further purified by gel filtration chromatography on a Sephadex G-50 column, eluting with mobile phase of $0.05 \mathrm{M} \mathrm{NH}_{4} \mathrm{HCO}_{3}$ (pH 8.0). The fractions containing the nanoparticleslabeled SA were collected and stored at $4{ }^{\circ} \mathrm{C}$ after diluting with $0.1 \mathrm{M}$ phosphate buffer $(\mathrm{pH}$ 7.4)containing $0.1 \%$ BSA, $0.05 \% \mathrm{NaN}_{3}$ and $0.9 \% \mathrm{NaCl}$.

\subsection{Coating biotinylated goat anti-mouseIgG to mi- crowell and fluorescent immunoassay}

To coat biotinylated goat anti-mouse IgG to microwell, $200 \mu \mathrm{L} 10 \mu \mathrm{g} / \mathrm{mL}$ of biotinylated goat anti-mouse IgG (in $0.5 \mathrm{M} \mathrm{NaHCO}_{3}, \mathrm{pH}$ 9.6) was added to each well of 96-microwells plates (black) and incubated for $24 \mathrm{~h}$ at $4{ }^{\circ} \mathrm{C}$. After twice washing with $0.05 \mathrm{M}$ phosphate buffer (pH 7.4, containing $0.9 \% \mathrm{NaCl}$ and $0.05 \%$ Tween-20) three times, the microwells were blocked by incubation with $2 \%$ BSA (w/v) for $2 \mathrm{~h}$ at room temperature.

To perform fluorescent immunoassay, a serial dilution of nanoparticles-labeled SA in $0.1 \mathrm{M}$ phosphate buffer (pH 7.0, containing $0.9 \% \mathrm{NaCl}$ and $0.1 \% \mathrm{BSA}$ ) was added to each well and let the plate incubated for 3h at $37{ }^{\circ} \mathrm{C}$. After excess nanoparticles-labeled SA was removed thoroughly by washing four times in $0.05 \mathrm{M}$ phosphate buffer $(\mathrm{pH} 7.4$, containing $0.9 \% \mathrm{NaCl}$ and $0.05 \%$ Tween-20) with vigorous shaking, fluorescence intensities were measured in a THERMO Multiskan Ascent.

\section{Results and Discussion}

\subsection{Characterization of the nanoparticles}

TEM imaging of the nanoparticles are shown in Fig.2. Results show that the nanoparticles are spherical, monodisperse, and uniform in shape and size with average diameter of $70 \pm 5 \mathrm{~nm}$. Black dots embedded inside the silica network can be observed due to the presence of heavy metal atom in the dye [12].

Absorption and fluorescence emission spectra of $\mathrm{Ru}(\mathrm{bpy})_{3}$ dye and the $\mathrm{Ru}(\mathrm{bpy})_{3}$-doped nanoparticles are shown in Fig.2A and Fig.2B. The absorption spectra of the pure $\mathrm{Ru}(\mathrm{bpy})_{3}$ dye and the nanoparticles display a same profile in aqueous solution. Their emission spectra also show a same profile but maximum emission wavelength of the nanoparticles shift $8 \mathrm{~nm}$, toward the shorter wavelength. 
nanobe.org

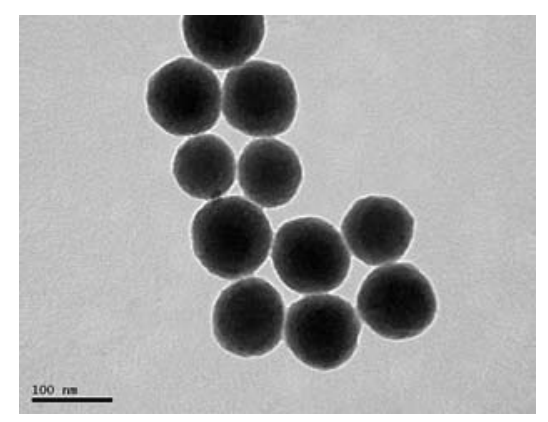

Figure 1. Transmission electron micrograph images of Ru(bpy)3-doped silica nanoparticles
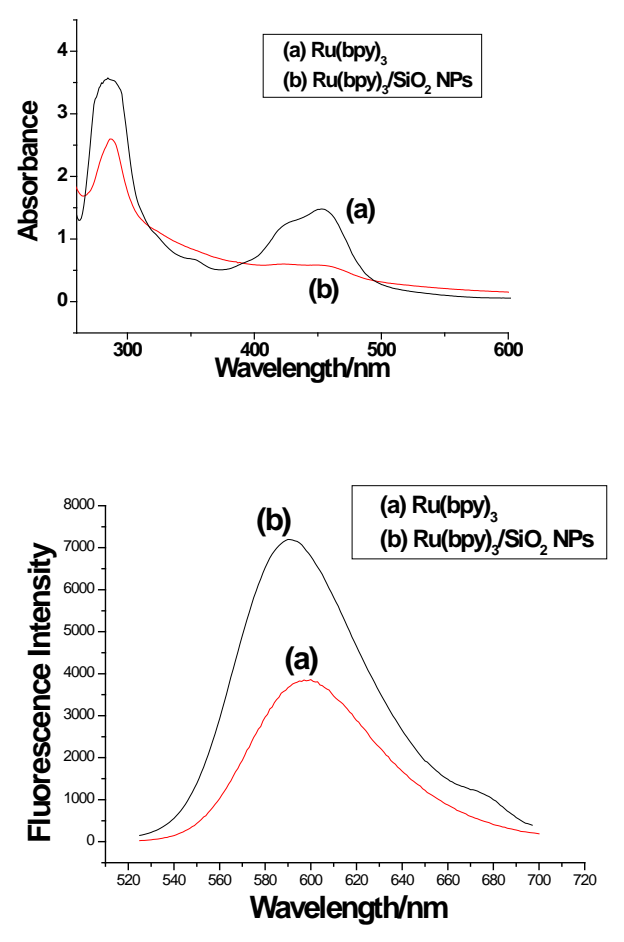

Figure 2. Absorption spectrum(A) and emission spectrum (B) of the Ru(bpy) $)_{3}$-doped nanoparticle and pure Ru(bpy)3

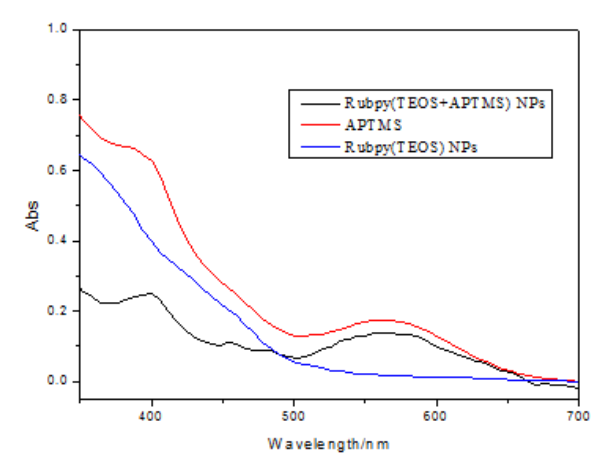

Figure 3. Results of confirmation of $-\mathrm{NH}_{2}$ groups on surface of the nanoparticles

\subsection{Confirmation of amino groups on surface of the nanoparticles}

When ninhydrin reacts with amino compound, a blue-violet compound is produced, which has a absorbance peak at about $570 \mathrm{~nm}$ [13]. Based on this fact, confirmation of amino groups on surface of the nanoparticles was investigated. Pure APTMS and two types

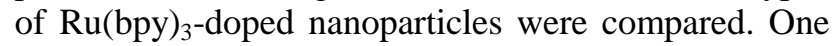
type of the nanoparticles was prepared through copolymerization of APTMS and TEOS, another type of the nanoparticles was prepared through polymerization of TEOS but without APTMS. As shown in Fig.4, pure APTMS and the former nanoparticles all have maximal absorbance peaks at 563nm, whereas the nanoparticles prepared without APTMS have non- absorbance peak at $563 \mathrm{~nm}$. The results demonstrated that amino groups had been introduced to the surface of the nanoparticles directly during the preparation process. Since the nanoparticles have active amino groups on their surface, they can be directly used to conjugate biological molecules without complicated surface modification.

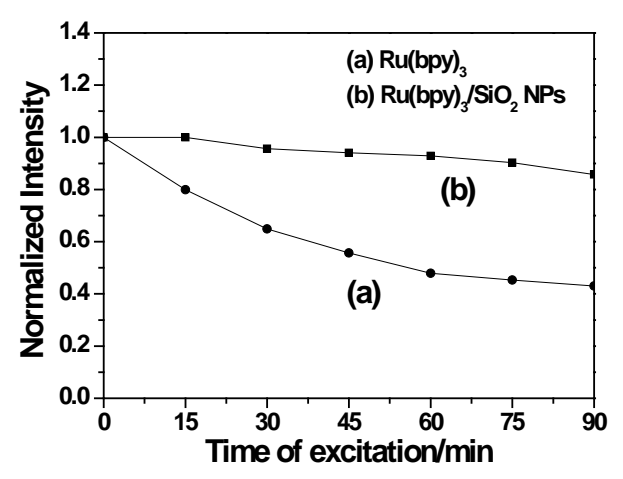

Figure 4. Results of photostability experiments of the nanoparticles

\subsection{Photostability and dyeleaking of the nanopar- ticles}

As shown in Figure 4, the fluorescent intensity of pure $\mathrm{Ru}(\mathrm{bpy})_{3}$ was decreased approximately $57 \%$ after 1.5h of continuous excitation, whereas the fluorescent intensity of the nanoparticles was only decreased $10 \%$. The high photostability of the nanoparticles is due to the fact that the $R u(b p y)_{3}$ dye in the nanoparticles is coated surroundingly by silica, which isolates the dye molecules from the outside environment such as solvent molecules, oxygen and free radicals caused by light exposure. Therefore, effectively protects the dye molecules from photodecomposition.

As shown in Figure 5, the fluorescent intensity of the nanoparticles was only decreased approximately $0.2 \%$ after continuously ultrasonic $60 \mathrm{~min}$ in aqueous solution. The results indicate the nanoparticles are stable in aqueous solution. 


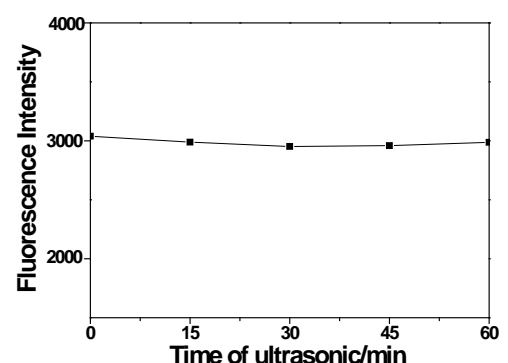

Figure 5. Results of dyeleaking experiments of the nanoparticles

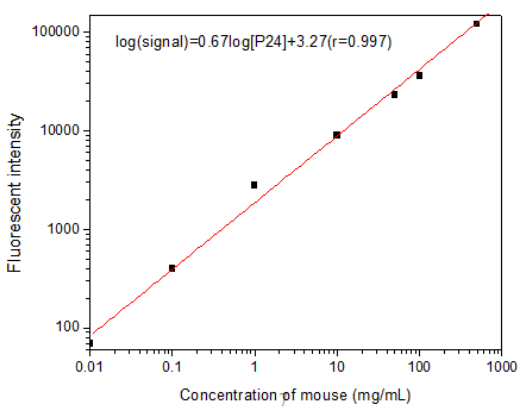

Figure 6. Results of fluorescent immunoassay using the nanoparticles as fluorescent probes.

\subsection{Application of the nanoparticles to fluorescent immunoassay}

This assay system is identical to that of ELISA except that fluorescent nanoparticles takes the place of enzyme. After the coated plates were blocked with BSA, the SA-nanoparticles as a probe was added to the 96-well microtiter plate. After unbound SAnanoparticles were washed away, the fluorescence intensities were measured. Results shown in Fig. 6 indicate a highly specific binding of the SA-nanoparticles to the biotinylated goat anti-mouse IgG. The relationship between the fluorescence intensities and the SAnanoparticles concentrations was linear in the experimental range. These results demonstrate the potential to apply this newly developed fluorescent nanoparticles in various sensitive immunoassay.

\section{Acknowledgements}

Financial support from the Shanghai Pujing Program, Shanghai Nano Program (No. 0752nm024), Nation 973(2010CB933901), and Shanghai Leading Academic Discipline Project (No. S30109) is gratefully acknowlegded.

\section{References}

1. Bagwe RP, Yang CY, "Optimization of Dye-Doped silica nanoparticles prepared using a reverse microemulsion me- thod”. Langmuir, vol. 20, pp. 8336-8342, April 2004. do $\mathrm{i}: 10.1021 /$ la 049137i

2. Tan WH, Wang KM, He XX, "Bio-nanotechnology based on silica nanoparticles”. Medicinal Research Reviews, vol. 24, pp. 621-638, April 2004. doi:10.1002/med.20003

3. Zhao XJ, Hilliard LR, Tan WH, “A rapid bioassay for single bacterial cell quantitation biotinylated goat antimouse-IgG were immobilized on 96-well micro-titer plates and using bioconjugated nanoparticles”. PNAS, vol. 101, pp. 15027-15032, October 2004. doi:10.1073/pnas.0404 806101

4. Zhao XJ, Dytioco RT, Tan WH, "Ultrasensitive DNA detection using highly fluorescent bioconjugated nanoparticles”. JACS, vol. 125, pp. 11474-11475, 2003 doi:10.1 021/ja0358854

5. Cui DX, Han YD, Li ZM, Song H, Wang K. "Fluorescent Magnetic Nanoprobes for in vivo Targeted Imaging and Hyperthermia Therapy of Prostate Cancer”. Nano Biomed. Eng. 2009,1, 61.

6. Cui DX, Li Q, Huang P, Wang K, Kong YF, Zhang H, You XG, He R, Song H, Wang JP, Bao CC, Asahi T, Gao F, Osaka T. "Real time PCR based on Fluorescent Quenching of Mercaptoacetic Acid-Modified CdTe Quantum Dots for Ultrasensitive Specific Detection of Nucleic Acids”. Nano Biomed. Eng.2010, 2(1),44.

7. Xie CJ, Yin DG, Li J, Zhang L. " Preparation of a Novel Amino Functionalized Fluorescein-doped Silica Nanoparticle for pH Probe”. Nano Biomed. Eng. 2009,1,27.

8. Yin DG, Zhang L, Xie CJ, Liu BH. "Preparation and Characterization of DPPDA-Eu ${ }^{3+}$ Doped Silica Fluorescent Nanoparticles” Nano Biomed. Eng. 2010, 2, 39.

9. Ye ZQ, Tan MQ, Wang GL, "Preparation, characterization, and timeresolved fluorometric application of silica-coated terbium(III)fluo-rescent nanoparticles”. Analytical Chemistry, vol. 76, pp.513-518, 2004 doi:10.1021/ac030177m

10. Santra S, Wang KM, Tan WH, "Development of novel dyedopped silica nanoparticles for biomarker application". Journal of Biomedical Optics, vol. 6, pp. 160-166, 2001 doi:10.1117/1.1353590

11. Wang L, Zhao WJ, "Fluorescent nanoparticles for multiplexed bacteria monitoring” Bioconjugate Cemistry, vol.18, pp. 297-301, 2007 doi:10.1021/bc060255n

12. Santra S, Wang KM, Tan WH, "Conjugation of biomolecules with luminophore-dopes silica nanoparticles for photostable biomarkers". Analytical Chemistry, vol. 73, pp. 4988-4993, 2001 doi:10.1021/ac010406+

13. Alyssa HC, Tutt TJ, Galloway M, "Surface modification of poly(methyl methacrylate) used in the fabrication of microanalytical Devices”. Analytical Chemistry, vol. 72, pp. 5331-5337, 2000 doi:10.1021/ac000685

Received 26 May, 2010; accepted 10 June, 2010; published online 26 June, 2010.

Copyright: (C) 2010 D. Yin et al. This is an open access article distributed under the terms of the Creative Commons Attribution License, which permits unrestricted use, distribution, and reproduction in any medium, provided the original author and source are credited. 